\title{
Delivery of Brief Interventions for Heavy Drinking in Primary Care: Outcomes of the ODHIN 5-Country Cluster Randomized Trial
}

Peter Anderson, PbD

Simon Coulton, PbD

Eileen Kaner, $\mathrm{PbD}$

Preben Bendtsen, PbD

Karolina Ktoda, $\mathrm{PbD}$

Jillian Reynolds, MSc

Lidia Segura, MSc

Marcin Wojnar, PbD

Artur Mierzecki, PbD

Paolo Deluca, PbD

Dorotby Newbury-Birch, PbD

Katbryn Parkinson, PbD

Katarzyna Okulicz-Kozaryn, PbD

Colin Drummond, MD

Antoni Gual, $\mathrm{PbD}$
Conflicts of interest: Dr Gual reports grants and personal fees from Lundbeck and D\&A Pharma, grants from Teva, and personal fees from AbbVie, outside the submitted work. The other authors report baving no conflicts of interest.

\section{CORRESPONDING AUTHOR}

Peter Anderson

Institute of Health and Society

Newcastle University

Baddiley-Clark Bldg, Richardson Rd

Newcastle upon Tyne

NE2 4AX, England

Peter.Anderson@newcastle.ac.uk

\begin{abstract}
PURPOSE We aimed to test whether 3 strategies-training and support, financial reimbursement, and an option to direct screen-positive patients to an Internetbased method of giving brief advice-have a longer-term effect on primary care clinicians' delivery of screening and advice to heavy drinkers operationalized with the Alcohol Use Disorders Identification Test-Consumption (AUDIT-C) tool.
\end{abstract}

METHODS We undertook a cluster randomized factorial trial with a 12-week implementation period in 120 primary health care units throughout Catalonia, England, Netherlands, Poland, and Sweden. Units were randomized to 8 groups: care as usual (control); training and support alone; financial reimbursement alone; electronic brief advice alone; paired combinations of these conditions; and all 3 combined. The primary outcome was the proportion of consulting adult patients (aged 18 years and older) receiving intervention-screening and, if screen-positive, advice-at 9 months.

RESULTS Based on the factorial design, the ratio of the log of the proportion of patients given intervention at the 9-month follow-up was 1.39 (95\% Cl, 1.03-1.88) in units that received training and support as compared with units that did not. Neither financial reimbursement nor directing screen-positive patients to electronic brief advice led to a higher proportion of patients receiving intervention.

CONCLUSIONS Training and support of primary health care units has a lasting, albeit small, impact on the proportion of adult patients given an alcohol intervention at 9 months.

Ann Fam Med 2017;15:335-340. https://doi.org/10.1370/afm.2051.

\section{INTRODUCTION}

$\mathrm{A}$ lcohol consumption is partly or entirely the cause of more than 200 diseases, injuries, and other health conditions with 3-digit International Classification of Diseases 10th Revision (ICD-10) codes, ${ }^{1}$ and alcohol is the sixth most important risk factor for ill health and premature death at the global level. ${ }^{2}$ Heavy drinkers who reduce consumption decrease their risk of mortality when compared with those who continue to drink heavily. ${ }^{3,4}$ Systematic reviews demonstrate that primary health care-based screening and brief advice programs are effective in reducing alcohol consumption and related harm. ${ }^{5-7}$

Many national and international guidelines recommend routine screening for heavy drinking in primary care and the offering of advice to screen-positive patients. ${ }^{8-10}$ In many settings, however, there is a large gap between need and provision of advice. Elsewhere, we have shown that only 11 per 1,000 eligible patients consulting their primary care clinician over a 4 -week period were screened for heavy drinking and, if screen-positive, subsequently advised to reduce their alcohol consumption (average across Catalonia, England, Netherlands, Poland, and Sweden). ${ }^{11}$ It is possible to increase the proportion of eligible patients screened and advised for heavy drinking. ${ }^{2}$ 
Previously, we have shown in the Optimizing Delivery of Healthcare Interventions (ODHIN) trial that providing training and support and financial reimbursement to primary care professionals increased the proportion of adult patients who received intervention for heavy drinking during a 12-week implementation period. Such interventions include screening and, if screen-positive, subsequent advice. The opportunity to refer patients to an electronically delivered brief advice program (hereafter simply called e-advice) did not change the proportion, however. ${ }^{13}$ During the implementation period, practitioners were asked to screen all consulting adult patients-regardless of the reason for their visit-for heavy drinking using the Alcohol Use Disorders Identification Test-Consumption (AUDIT-C) too ${ }^{14}$ and to give brief advice to screenpositive patients. For the primary care physicians, training and support, and financial reimbursement worked mainly by increasing the proportion of patients screened, rather than by increasing the proportion of screen-positive patients advised, which was already high (74\%) at baseline.

In this follow-up study, we investigated the impact of these 3 strategies 6 months later, at the 9 -month follow-up. We hypothesized that primary health care units that had received training and support would maintain a higher proportion of patients receiving intervention; that units that had received financial reimbursement would revert to their baseline level once that incentive was gone; and that units giving e-advice, which did not yield improvement during the implementation period, would similarly not show any gain with longer follow-up.

\section{METHODS}

\section{Design}

The ODHIN trial had a cluster randomized $2 \times 2 \times 2$ factorial design ${ }^{15}$ (with no trial deviations) allowing us to study the impact of the 3 implementation strategies on primary health care units' screening and provision of advice for heavy drinking, operationalized by AUDIT-C. ${ }^{14}$ Data were collected between August 2012 and December 2013. The trial flow is shown in Figure 1.

\section{Participants}

Primary health care units with approximately 5,000 to 20,000 registered patients from 5 countries (Catalonia in Spain, England, Netherlands, Poland, and Sweden) were the unit of randomization, implementation, and analysis. Participating units were volunteers drawn from administrative or academic registries of primary health care units at the country level or the withincountry regional level.

\section{Implementation Strategies}

Units were recruited between September 2012 and May 2013. After they gave formal agreement to participate in the trial, a 4 -week baseline measurement period took place. Two to 6 weeks later, the 12-week implementation period occurred, with the start date for each country occurring between November 2012 and July 2013.

The trial had 8 groups: a control group and 7 experimental groups that tested the implementation strategies alone and in combinations (Figure 1). ${ }^{3}$ All of the experimental groups received the same input as the control group, but with additional components.

Units in the control group were given a package containing a summary card of their country's guideline recommendations for screening and advice for hazardous and harmful alcohol consumption, without demonstration. They were also given instructions on how to complete the trial record sheet.

The 3 implementation strategies tested explored different ways of increasing screening and advice for heavy drinking. For the training and support strategy, units were offered 2 initial 1- to 2 -hour face-to-face

Figure 1. Flow chart of primary health care units in the trial.

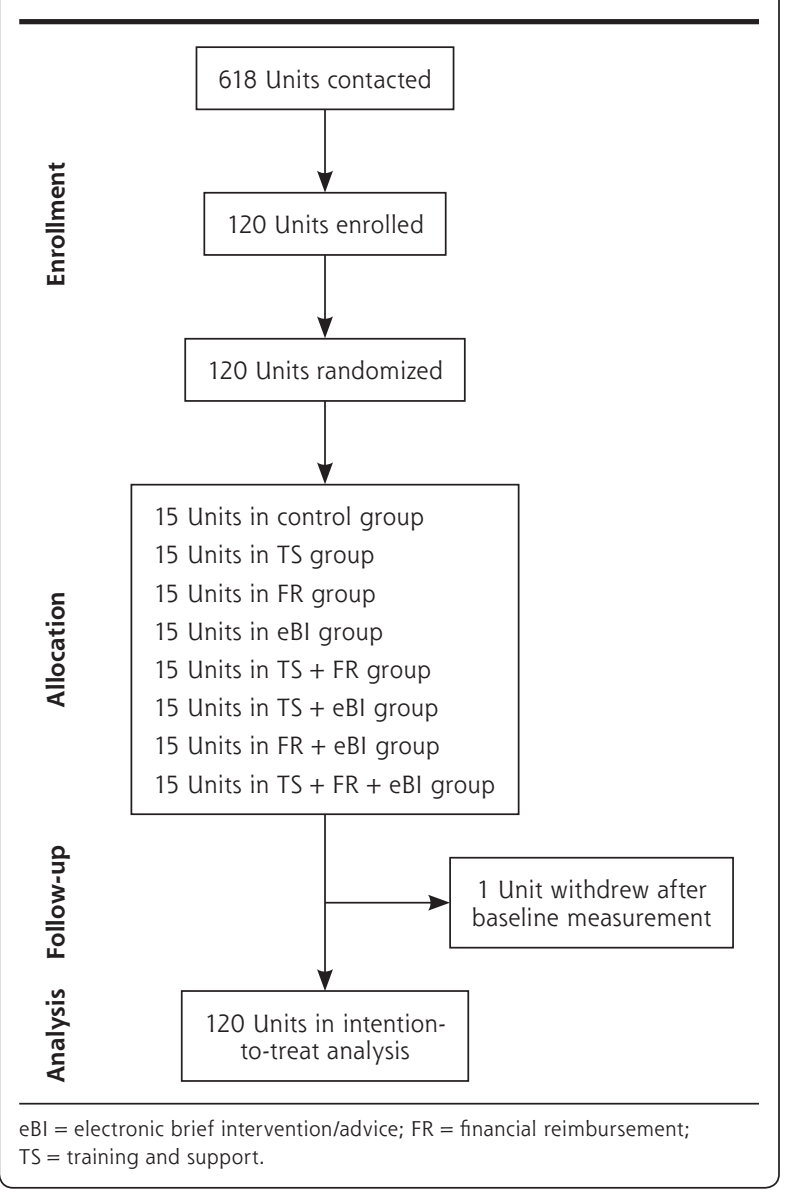


educational training sessions, and 1 telephone support call lasting 10 to 30 minutes with the lead unit contact person during the first 4 to 6 weeks of the implementation period. For the financial reimbursement strategy, units were paid for screening and advice activities during the implementation period. For the e-advice strategy, units were asked to direct identified screenpositive patients to an approved digital brief intervention advice program, by giving them a printed leaflet that included the website address and briefly explaining why they should log on to the site and how to use it.

Each strategy was tested both alone and in paired combination with each of the others; additionally, all 3 strategies were tested together (Figure 1). In groups assigned to the financial reimbursement plus e-advice strategies, units were given the reimbursement even if clinicians only referred screen-positive patients to e-advice.

Participating units were asked to screen all adult patients (aged 18 years and older) who consulted the unit using AUDIT-C. Based on country guidelines, screen-positive patients were defined in Catalonia and England as men and women who scored at least 5 on this tool, and in Poland, the Netherlands, and Sweden as men who scored at least 5 and women who scored at least 4 . All units were asked to deliver brief advice of 5 to 15 minutes' duration to screen-positive patients, with the length and format of the advice based on country-specific guidelines.

The 12-week implementation period was followed by a 6 -month washout period, during which training and support, and financial reimbursement were discontinued, but clinicians in the e-advice groups were still free to direct screen-positive patients to that package. At the end of this period, we undertook a 4 -week follow-up measurement period (9-month follow-up).

\section{Outcomes}

Screening and brief advice activity was measured during the baseline period (which lasted 4 weeks), the implementation period (12 weeks), and the 9-month follow-up period (4 weeks). This activity was measured using paper tally sheets, or electronic records in the case of Catalonia, completed by the clinicians. The tally sheets included AUDIT-C questions, AUDIT-C scores, and check boxes to indicate the method of delivering advice to each patient: oral advice, an advice leaflet, referral to the e-advice program, or referral to another clinician in or outside the unit for advice. The primary outcome measure was the proportion of consulting adult patients receiving intervention (screening and, if screen-positive, advice) per unit, defined as the number of AUDIT-C-positive patients who received 1 or more of oral advice, an advice leaflet, referral to the e-advice program, or referral to another clinician for advice, divided by the total number of adult consultations of the participating clinicians per unit.

\section{Randomization and Blinding}

Randomization of the primary health care units took place after they formally agreed to take part in the trial. They were randomly allocated to 1 of the 8 groups by the ODHIN coordinating center, the Hospital Clinic of Barcelona, using Excel (Microsoft Corp). Stratified randomization by country was used to ensure equal numbers of 15 units per group, with equal numbers per group per country ( 3 units per group per country). The country-based research teams were informed of the allocation after baseline data had been collected, and those teams in turn informed the units.

\section{Sample Size}

We estimated that 56 primary health care units $(7$ per each of the 8 allocation groups) with a minimum of 1,000 adult patients consulting per month would be needed for an $80 \%$ chance of detecting an increase in the proportion of consulting adult patients given the intervention from $4 \%$ to at least $6 \%(\alpha=.05)$. In calculating our sample size, we used an estimate of an intraclass correlation coefficient (ICC) of 0.029 across primary care interventions ${ }_{1}^{16}$ based on a study of implementation of alcohol screening and advice in such units. ${ }^{17}$ As little information was available to guide selection of the ICC, we conducted a post hoc ICC calculation based on the proportion of patients receiving the intervention by individual primary health care clinicians across units, with an ICC at baseline of 0.03 , and a $95 \% \mathrm{CI}$ of 0.007 to 0.053 , including the estimate of 0.029 that was used. Sample size estimation was determined using Stata 12 (StataCorp LP). As country was used as the stratification criterion, each country included a minimum of 24 primary health care units.

\section{Statistical Methods}

Eighteen of the 120 units did not provide adequate data to calculate proportions at the 9-month follow-up; for these units, we used the proportions from the baseline measurement period. We undertook a sensitivity analysis excluding these 18 units to assess the impact of our assumption and found it to be valid. Distributional assumptions of the primary outcome were assessed, and natural log transformations were undertaken where appropriate. As this approach creates some issues with outcomes having a zero value, 0.001 was added to each proportion before log transformation. The primary outcome was analyzed in a generalized linear model with proportion screened and advised in the baseline period incorporated as a covariate. As the study is hierarchical 
in nature with primary health care units nested within countries, we used a multilevel approach, using country with random intercepts and slopes.

The study had a factorial design, whereby factors were coded with -1 and +1 , resulting in regression coefficients having one-half the effects. The factorial design is based on the premise that the effect of, for example, training and support instead of no training and support can be estimated not only from training and support vs control, but also from training and support plus financial reimbursement vs financial reimbursement alone, and so on for each combination, giving a pooled estimate with more precision. The factors for the interventions were therefore coded as follows:

$\mathrm{TS}=-1$ for control, FR, eBI, FR $+\mathrm{eBI}$ and,+1 for $\mathrm{TS}, \mathrm{TS}+\mathrm{FR}, \mathrm{TS}+\mathrm{eBI}, \mathrm{TS}+\mathrm{FR}+\mathrm{eBI}$

$\mathrm{FR}=-1$ for control, TS, eBI, TS $+\mathrm{eBI}$; and +1 for $F R, F R+T S, F R+e B I, F R+T S+e B I$

$\mathrm{eBI}=-1$ for control, FR, TS, TS $+\mathrm{FR}$ and +1 for $\mathrm{eBI}, \mathrm{eBI}+\mathrm{TS}, \mathrm{eBI}+\mathrm{FR}, \mathrm{eBI}+\mathrm{TS}+\mathrm{FR}$

where TS indicates training and support, FR indicates financial reimbursement, and eBI indicates electronic brief intervention/advice.

Factors were analyzed with exploration for potential interactions using a step-up approach and interpreted using the $R^{2}$ statistic. Where interactions were identified, we incorporated them into the regression equation. Analysis was conducted using Stata 14 (StataCorp LP) and MLwiN 2.02 (Centre for Multilevel Modelling, University of Bristol).

\section{RESULTS}

The mean number of patients registered in each primary health care unit was 10,000. On average, each unit had 1,500 adult consultations during the 4 -week baseline period. The mean age of patients was 53 years $(\mathrm{SD}=6)$, and $55 \%$ were men. Overall, $55 \%$ of participating clinicians per unit were physicians, 38\% were nurses, and 7\% were practice assistants. The mean age of participating clinicians per unit was 47 years $(\mathrm{SD}=5)$, and $74 \%$ were women

During the baseline period, the mean proportion of consulting adults given the intervention per unit across all 8 groups was 11.1 per 1,000 (95\% CI, 9.0-13.3). Table 1 shows proportions given the intervention for this baseline period, as well as the 12-week implementation and 9-month follow-up periods, by allocation group.
On the basis of the factorial design, the training and support implementation strategy had a significant positive effect on the proportion of consulting adult patients given intervention at the 9-month follow-up: the ratio of the log of the proportion given intervention was 1.39 (95\% CI, 1.03-1.88) for units that received training and support vs units that did not receive it (Table 2). None of the other strategies or combinations of strategies had a significant positive effect.

\section{DISCUSSION}

\section{Overall Findings}

Previously in the ODHIN trial, we showed that up to 4 hours of training and support for primary health care clinicians and financial reimbursement delivered during a 12 -week implementation period alone and in combination increased the proportion of heavy drinkers given an intervention (screening and advice given to screen-positive patients) to reduce their drinking. ${ }^{13}$ During that implementation period, the ratio of the $\log$ of the proportion given the intervention was 1.61 (95\% CI, 1.24-2.10) in units that received training and support vs units that did not; for financial reimbursement, the ratio was 2.00 (95\% CI, 1.49-2.47), and for training and support plus financial reimbursement, the ratio was 2.44 (95\% CI, 1.85-3.22). In the present analysis, we have demonstrated a lasting effect of training and support at 9 months of follow-up, with the ratio of the log of the proportion given an intervention at that time of 1.39 (95\% CI, 1.03-1.88) for units that received training and support vs units that did not. We found no lasting impact of financial reimbursement.

The absolute impact of training and support was small. At baseline, 11.1 per 1,000 (95\% CI, 9.0-13.3)

\begin{tabular}{|c|c|c|c|}
\hline \multirow[b]{2}{*}{ Group } & \multicolumn{3}{|c|}{$\begin{array}{l}\text { Mean Proportion (SE) of Consulting Adult } \\
\text { Patients (per } 1,000) \text { Given Intervention }{ }^{a}\end{array}$} \\
\hline & Baseline & $\begin{array}{c}\text { 12-Week } \\
\text { Implementation }\end{array}$ & $\begin{array}{l}\text { 9-Month } \\
\text { Follow-up }\end{array}$ \\
\hline Control & 11.65 (3.29) & $5.35(1.16)$ & $5.98(1.55)$ \\
\hline TS & $13.94(4.22)$ & $11.72(3.37)$ & $8.40(2.12)$ \\
\hline FR & $9.82(1.65)$ & $17.97(4.44)$ & $10.00(5.45)$ \\
\hline eBI & $15.63(3.42)$ & 9.89 (1.95) & 7.69 (2.04) \\
\hline $\mathrm{TS}+\mathrm{FR}$ & $10.87(3.03)$ & 32.07 (8.61) & $11.80(3.46)$ \\
\hline $\mathrm{TS}+\mathrm{eBI}$ & $9.39(2.54)$ & $9.22(1.99)$ & $7.64(1.88)$ \\
\hline$F R+e B I$ & $11.28(3.72)$ & $8.42(1.82)$ & $4.71(1.98)$ \\
\hline $\mathrm{TS}+\mathrm{FR}+\mathrm{eBI}$ & $6.59(1.63)$ & $16.91(3.05)$ & $9.74(2.70)$ \\
\hline
\end{tabular}


Table 2. Mean Natural Log of Proportion of Consulting Adult Patients Given Intervention at 9-Month Follow-up per Unit, by Presence or Absence of Factor

\begin{tabular}{|c|c|c|c|c|}
\hline Factor & $\begin{array}{c}\text { Mean Natural Log } \\
\text { of Proportion } \\
(95 \% \mathrm{Cl})\end{array}$ & $\begin{array}{c}\text { Mean Difference in } \\
\text { Natural Log of } \\
\text { Proportion vs Absent } \\
(95 \% \mathrm{Cl})\end{array}$ & $\begin{array}{l}\text { Ratio of Natural Log } \\
\text { of Proportion vs Absent } \\
(95 \% \mathrm{Cl})\end{array}$ & $P$ Value \\
\hline TS absent & $-5.399(-5.851$ to -4.947$)$ & - & 1.00 & \\
\hline TS present & $-5.070(-5.522$ to -4.618$)$ & $0.329(0.025$ to 0.634$)$ & 1.39 (1.03 to 1.88$)$ & .03 \\
\hline FR absent & $-5.269(-5.728$ to -4.810$)$ & - & 1.00 & \\
\hline FR present & $-5.200(-5.660$ to -4.741$)$ & $0.069(-0.241$ to 0.379$)$ & 1.07 (0.78 to 1.46$)$ & .66 \\
\hline eBI absent & $-5.253(-5.714$ to -4.792$)$ & - & 1.00 & \\
\hline eBI present & $-5.216(-5.677$ to -4.756$)$ & $0.037(-0.273$ to 0.346$)$ & 1.04 (0.76 to 1.41$)$ & .82 \\
\hline TS + FR absent & $-5.320(-5.772$ to -4.869$)$ & - & 1.00 & \\
\hline TS + FR present & $-4.977(-5.454$ to -4.499$)$ & $0.343(-0.010$ to 0.697$)$ & 1.41 (0.99 to 2.01$)$ & .06 \\
\hline $\mathrm{TS}+\mathrm{eBI}$ absent & $-5.209(-5.674$ to -4.744$)$ & - & 1.00 & \\
\hline $\mathrm{TS}+\mathrm{eBI}$ present & $-5.311(-5.801$ to -4.821$)$ & $-0.101(-0.460$ to 0.268$)$ & 0.90 (0.63 to 1.31$)$ & .58 \\
\hline $\mathrm{FR}+\mathrm{eBI}$ absent & $-5.314(-5.765$ to -4.863$)$ & - & 1.00 & \\
\hline $\mathrm{FR}+\mathrm{eBI}$ present & $-4.995(-5.473$ to -4.518$)$ & $0.319(-0.036$ to 0.674$)$ & 1.38 (0.96 to 1.96$)$ & .08 \\
\hline $\mathrm{TS}+\mathrm{FR}+\mathrm{eBI}$ absent & $-5.282(-5.737$ to -4.827$)$ & - & 1.00 & \\
\hline $\mathrm{TS}+\mathrm{FR}+\mathrm{eBI}$ present & $-4.899(-5.453$ to -4.345$)$ & $0.383(-0.081$ to 0.847$)$ & 1.47 (0.92 to 2.33$)$ & .11 \\
\hline
\end{tabular}

consulting adults were given the intervention. At the 9-month follow-up, the value dropped to 7.0 per 1,000 (95\% CI, 4.1-9.9) without and 9.4 per $1,000(95 \% \mathrm{CI}$, 6.8-12.0) with training and support. Training and support therefore attenuated the reduction in this activity over time.

\section{Strengths and Weaknesses}

One study strength was its factorial design, which ensured we had sufficient power to detect small changes with a relatively small number of primary health care units, just 24, per country. Another strength is that it was conducted across 5 countries with differing cultures and health system financing and management structures.

One study weakness is that participating units were volunteers drawn from administrative or academic registries until the required sample size was achieved. Data were not available to indicate whether these units were representative of those in the 5 countries as a whole. Previously, we found some evidence that the volunteering units were more motivated to work with drinkers than similar units from the same country in general. ${ }^{13} \mathrm{~A}$ second weakness of our study is that the tally sheet used to measure AUDIT-C included all of the options for giving advice. In itself, this sheet is an organizational intervention to support clinician behavior, although it was provided equally across all 8 groups. The tally sheet was completed by the clinician, and we did not perform any independent check to determine whether the advice was actually carried out, or to ascertain its fidelity in terms of content, length, and quality of the advice.

\section{Implications for Service Commissioners and Policy Makers}

On the basis of our ODHIN findings reported previously and here, we recommend that all jurisdictions consider providing training and support to primary health care clinicians in delivering screening and brief advice, so as to increase the provision of brief interventions delivered to patients in primary care who drink heavily. Given the drop-off in impact over time, repetition of training and support over a longer period is desirable. Our findings confirm some of the difficulties of the sustainability of financial reimbursement, as we found that its impact ceased when payments ended.

To read or post commentaries in response to this article, see it online at http://www.AnnFamMed.org/content/15/4/335.

Key words: primary health care; heavy drinking; implementation study; training and support; financial reimbursement; electronic brief intervention; practice-based research

Submitted August 23, 2016; submitted, revised, January 2, 2017; accepted January 11, 2017.

Author affiliations: Institute of Health and Society, Newcastle University, Newcastle, England (Anderson, Kaner, Parkinson); Department of Family Medicine, Maastricht University, Maastricht, the Netherlands (Anderson); Centre for Health Service Studies, University of Kent, Canterbury, England (Coulton); Department of Medical Specialist and Department of Medicine and Health, Linköping University, Motala, Sweden (Bendtsen); Independent Laboratory of Family Physician Education, Pomeranian Medical University, Szczecin, Poland (Kłoda, Mierzecki); Psychiatry Department, Neurosciences Institute, Hospital Clínic, IDIBAPS, Barcelona, Spain (Reynolds, Gual); Program on Substance Abuse, Public Health Agency, Government of Catalonia, Barcelona, Spain (Segura); Department of Psychiatry, Medical University of Warsaw, Warsaw, Poland 
(Wojnar); National Addiction Centre, Institute of Psychiatry, King's College London, London, England (Deluca, Drummond); Health and Social Care Institute, Teesside University, Middesbrough, United Kingdom (Newbury-Birch); State Agency for Prevention of Alcohol-Related Problems, Warsaw, Poland (Okulicz-Kozaryn); National Institute for Health Research Biomedical Research Centre for Mental Health, South London and Maudsley NHS Foundation Trust, London, England (Drummond).

Author contributions: All authors except P.A. and S.C. were involved in implementing the trial in their jurisdictions. S.C. and P.A. undertook analyses. P.A. wrote drafts of the manuscript. All other authors revised the manuscript critically. All authors read and approved the final manuscript.

Funding support: The research leading to these results or outcomes has received funding from the European Union's Seventh Framework Programme for Research, Technological Development, and Demonstration under grant agreement no. 259268: Optimizing Delivery of Healthcare Interventions (ODHIN). Organizations participating in ODHIN can be seen at www.odhinproject.eu. Radboud University Medical Center received cofunding from The Netherlands Organisation for Health Research and Development (ZonMW, Prevention Program), under grant agreement no. 200310017 - ODHIN - Optimizing Delivery of Healthcare Interventions in the Netherlands, according to Art.II.17 of the FP7 EC Grant Agreement. Colin Drummond is partly funded by the NIHR Biomedical Research Centre for Mental Health at South London and Maudsley NHS Foundation Trust and King's College London and partly funded by the NIHR Collaborations for Leadership in Applied Health Research and Care South London at King's College Hospital NHS Foundation Trust. Both Medical University of Warsaw and Pomeranian Medical University in Szczecin received cofunding for the ODHIN project from the Polish science financial resources in the years 2011-2014.

Disclaimer: Views expressed are those of the author(s) and not necessarily those of NHS, NIHR, Department of Health, or European Commission.

Trial registration: ClinicalTrials.gov. Trial identifier: NCT01501552.

Acknowledgments: We thank all participating primary health care units and practitioners for their support with the trial. We thank Steven Teerenstra (statistician, at the Department Health Evidence Radboud University Medical (enter) who assisted P.A. in designing the analytic plan for the factorial randomized controlled trial design. We thank Birgit Jansen (research assistant, Scientific Center for Quality of Healthcare [IQ Healthcare], Radboud University Medical Center) and Paddy Hinsssen (research assistant, Faculty of Health, Medicine and Life Sciences, Maastricht University) for executing many logistic procedures to gather the tally sheets. We thank the Catalan Society of Family and Community Medicine, the Catalan Association of Family and Community Nursing, and the Alcohol Network of Referents on Alcohol (XaROH) of the Programa Beveu Menys, who collaborated in the implementation of the trial in Catalonia. We thank Sebastián Calero from the Catalan Health Institute's Healthcare Issues' Area of Clinical Development; Carmen Olmos and Manuel Iglesias from the eCAP Functional Competences Centre; and Manuel Medina, Francesc Fina, Leonardo Méndez, and Eduard Hermosillo from the Information System for Research Development in Primary Health Care (SIDIAP database) for supporting the Catalan ODHIN team in the adaptation of the electronic medical records (eCAP) and in the data extraction for the trial. The following individuals were part of the WP5 ODHIN group, in addition to the authors of this article: Miranda Laurant (Netherlands), Myrna Keurhorst (Netherlands), Trudy van der Weijden (Netherlands), and Michel Wensing (Netherlands). E.K. was supported via membership of the NIHR School of Public Health Research and NIHR School of Primary Care Research.

CONSORT statement: This article adheres to the CONSORT (Consolidated Standards of Reporting Trials) 2010 guidelines..$^{18}$

\section{References}

1. World Health Organization. Global Status Report on Alcohol and Health, 2014. Geneva, Switzerland: World Health Organization; 2014.

2. Forouzanfar MH, Alexander L, Anderson HR, et al; GBD 2013 Risk Factors Collaborators. Global, regional, and national comparative risk assessment of 79 behavioural, environmental and occupational, and metabolic risks or clusters of risks in 188 countries, 1990-2013: a systematic analysis for the Global Burden of Disease Study 2013. Lancet. 2015;386(10010):2287-2323.

3. Fillmore KM, Kerr WC, Bostrom A. Changes in drinking status, serious illness and mortality. J Stud Alcohol. 2003;64(2):278-285.

4. Rehm J, Roerecke M. Reduction of drinking in problem drinkers and all-cause mortality. Alcohol Alcohol. 2013;48(4):509-513.

5. Kaner EF, Beyer F, Dickinson HO, et al. Effectiveness of brief alcohol interventions in primary care populations. Cochrane Database Syst Rev. 2007;18(2):CD004148.

6. Jonas DE, Garbutt JC, Amick HR, et al. Behavioral counseling after screening for alcohol misuse in primary care: a systematic review and meta-analysis for the U.S. Preventive Services Task Force. Ann Intern Med. 2012;157(9):645-654.

7. O'Donnell A, Anderson P, Newbury-Birch D, et al. The impact of brief alcohol interventions in primary healthcare: a systematic review of reviews. Alcohol Alcohol. 2014;49(1):66-78.

8. National Institute for Health and Clinical Excellence. Alcohol-use disorders: prevention. NICE. https://www.nice.org.uk/guidance/ph2 4? unlid =15956226520162272344152010. Published Jun 2010. Accessed Aug 1, 2016.

9. Dua T, Barbui C, Clark N, et al. Evidence-based guidelines for mental, neurological, and substance use disorders in low- and middleincome countries: summary of WHO recommendations. PLOS Med. 2011;8(11):e1001122.

10. Health Scotland. Alcohol Advice 2012/2013. https://isdscotland.scot. nhs.uk/Health-Topics/Drugs-and-Alcohol-Misuse/Publications/201306-25/2013-06-25-ABI-Report.pdf?89017885924. Published Jun 25, 2013. Accessed Aug 1, 2016.

11. Bendtsen $P$, Anderson $P$, Wojnar M, et al. Professional's attitudes do not influence screening and brief intervention rates for hazardous and harmful drinkers: results from ODHIN study. Alcohol Alcohol. 2015;50(4):430-437.

12. Keurhorst M, van de Glind I, Bitarello do Amaral-Sabadini M, et al. Implementation strategies to enhance management of heavy alcohol consumption in primary health care: a meta-analysis. Addiction. 2015;110(12):1877-1900.

13. Anderson P, Bendtsen P, Spak F, et al. Improving the delivery of brief interventions for heavy drinking in primary health care: outcome results of the ODHIN five country cluster randomized factorial trial. Addiction. 2016;111(11):1935-1945.

14. Jonas DE, Garbutt JC, Brown JM, et al. Screening, Behavioral Counseling, and Referral in Primary Care to Reduce Alcohol Misuse. Comparative Effectiveness Reviews, No. 64. Rockville, MD: Agency for Healthcare Research and Quality; 2012.

15. Keurhorst MN, Anderson P, Spak F, et al. Implementing training and support, financial reimbursement, and referral to an Internet-based brief advice program to improve the early identification of hazardous and harmful alcohol consumption in primary care (ODHIN): study protocol for a cluster randomized factorial trial. Implement Sci. 2013;8:11.

16. Adams G, Gulliford MC, Ukoumunne OC, Eldridge S, Chinn S, Campbell MJ. Patterns of intra-cluster correlation from primary care research to inform study design and analysis. J Clin Epidemiol. 2004;57(8):785-794.

17. Kaner EFS, Lock CA, McAvoy BR, Heather N, Gilvarry E. A RCT of three training and support strategies to encourage implementation of screening and brief alcohol intervention by general practitioners. BrJ Gen Pract. 1999;49(446):699-703.

18. Moher D, Hopewell S, Schulz KF, et al. CONSORT 2010 explanation and elaboration: updated guidelines for reporting parallel group randomised trials. BMJ. 2010;340:c869. 